SELECTED PAPER AT NCSP'17

\title{
Preliminary Verification of Spatial Perception Support for Tele-Existence Robot Manipulation
}

\author{
Noritaka Funada and Satoshi Suzuki \\ Graduate School of Engineering, Tokyo Denki University \\ 5 Asahi-cho, Adachi-ku, Tokyo 120-8551, Japan \\ E-mail: funadan@mail.suzulab.fr.dendai.ac.jp, ssuzuki@fr.dendai.ac.jp
}

\begin{abstract}
For a general tele-existence robot (TER), it is difficult for the operator to recognize the circumstances around the TER by observing the narrow view from the camera. To overcome this difficulty, we focused on a new method that provides two-dimensional (2D) map based on a laser range finder (LRF) in addition to the camera view. Existing simple 2D map methods have not been sufficiently considered from the view point of the operator's spatial perception; hence, in this study, a new remote manipulation system providing a real-time blurred 2D map is presented. Next, the operator's spatial perception using the $2 \mathrm{D}$ map was investigated through a tele-operation experiment by changing the scan area of the LRF and the accumulated time used to make motion blur on the 2D map. The conditions employed to make the blurred 2D map were changed among nine cases combining three scan ranges and three accumulated times. The spatial perception was also investigated by analyzing distances measured by the LRF. By comparing three distance data groups using the Kruskal-Wallis test, significant differences in the minimum distance were confirmed when the scan ranges were 1,3 and $5 \mathrm{~m}$ $(p<.001)$. Also, significant differences in the distance were confirmed when the accumulated times were $0.1,0.3$ and $0.5 \mathrm{~s}(p<.001)$. Therefore, it was confirmed that the operator's spatial perception was improved by increasing the scan range and the motion blur using the 2D map.
\end{abstract}

\section{Introduction}

A tele-existence robot (TER) can be manipulated from remote locations with a PC via the Internet. Since TERs are equipped with a video conference system, the user can effectively have face-to-face communication with a person at a remote locations. On the other hand, in order to manipulate a TER at the remote locations without collision with obstacles, the TER operator has to recognize the positional relationship between the remote robot and the obstacles around it. For a general TER, the operator has to recognize the environment around the robot from transmitted visual information that is monitored by a single camera mounted on the TER; however, it is difficult to operate the TER on the basis or limited information due to the narrow angle of view [1] and the poor depth perception [2].

To overcome this difficulty, previous studies utilized a head-mounted display (HMD) [3], and real-time map navigation using a laser range finder (LRF) [4]. HMD has the advantage that the operator can recognize distance and depth perception owing to the three-dimensional (3D) effect. However, it is not suitable for the TER operation, for which the aim is natural face-to-face communication. On the other hand, a motion parallax is required to overcome the poor depth perception; however, motion parallax cannot function because of the short distance if the space where the TER is used is small, such as an office. This is because in remote manipulation, the operator's perception of short distances is affected by the perception of depth related to the body schema (BS), which is unconsciously defined as a postural model of the body [5],[6]. A two-dimensional (2D) map rigorously gives the current distance between the TER and [5],[6] objects; thus, 2D map navigation using the LRF will support the operator's spatial perception. Moreover, the involvement of the operator will increase because it can provide spatial information around the TER; therefore, it will also modify the BS.

Thus, we focused on a new method providing a 2D map based on LRF information in addition to the ordinary camera view. Existing simple 2D map methods were insufficient because the appropriate conditions for providing adequate map information were not sufficiently considered.

To improve the performance of the 2D map, the following two factors have to be taken into consideration for the feasible design of a sophisticated TER manipulation system:

Factor 1: The scan range on the 2D map

Factor 2: The accumulated time used to make motion blur on the 2D map

Factor 1 is focused on since the range displaying the map 
affects the spatial perception [7]; for example, if the scan range of the 2D map is large, it may be difficult to notice dangerous situations. Factor 2 is also focused on since a person can easily perceive motion when motion blur is added to images [8]; for example, if the motion blur used to display the 2D map is increased, the user will easily recognize the circumstances around the TER.

In this study, a new remote manipulation system providing a real-time blurred 2D map is presented. Moreover, the operator's spatial perception using the 2D map was investigated through a tele-operation experiment by changing two factors, operator's spatial perception was investigated by analyzing same scan range group and same accumulated time group concerning distances measured by the LRF during the TER manipulation.

\section{System Configuration of Tele-Operation System}

In this study, we built a tele-operation system for a teleoperation experiment to enhance special perception. The system provides the operator with a $2 \mathrm{D}$ map, where the scan range can be changed to three values $(1,3$ and $5 \mathrm{~m})$ to investigate factor 1 . This system can also change the accumulated time used to produce a blur effect on the 2D map to $0.1,0.3$ and $0.5 \mathrm{~s}$ to investigate factor 2 . Figure 1 shows the configuration of the tele-operation system.

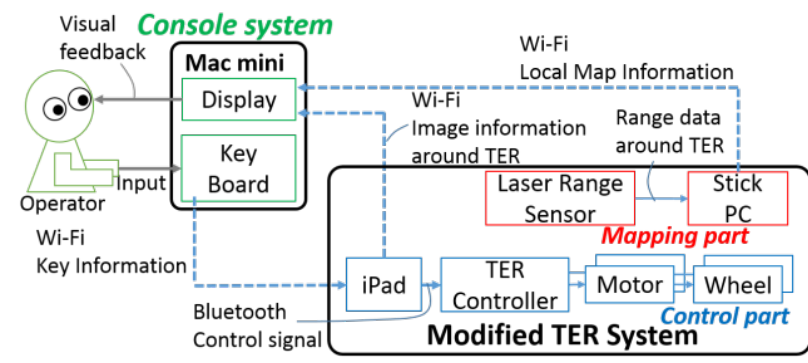

Figure 1: Configuration of tele-operation system

A mobile robot "Double" (Double Robotics Inc.) was utilized. The system was built with a Mac mini, an iPad, an LRF (URG-04LX-UG01, Hokuyo Automatic Co., Ltd.) and a stick PC (CSTK-32W, Intel Co., Ltd.). Each computer communicates via Wi-Fi. The command from the iPad to Double was translated by Bluetooth. The role of each computer is as follows:

- The Mac mini sends commands (keyboard inputs) from the operator to the iPad, and it shows a camera view and a 2D map on the display of the console.

- The iPad sends commands for speed control to Double based on the commands from the Mac mini. It sends images captured by the iPad to the Mac mini.
- The stick PC sends images of the 2D map that is drawn on the basis of the range data to the Mac mini. It logs data measured by the LRF.

Figure 2(a) shows the TER customized for our experiment and Fig.2(b) shows the console system used for teleoperation.
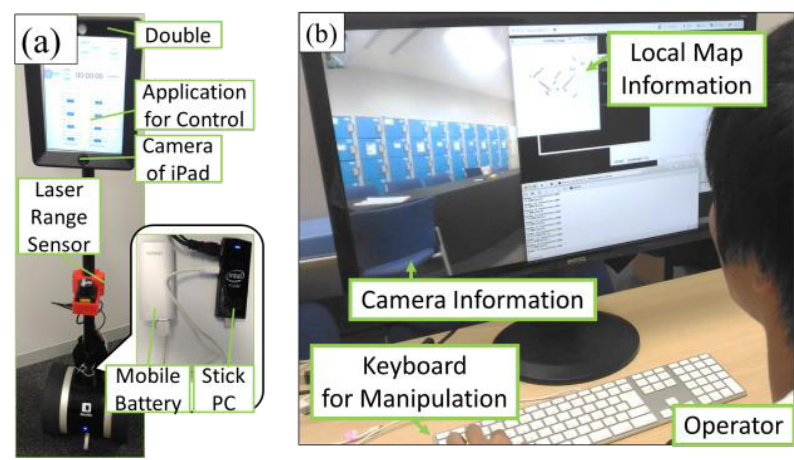

Figure 2: Tele-operation system for remote experiment:

(a) TER, (b) Console system for tele-operation

Figure 3 shows examples of blurred 2D maps when the scan ranges are 1, 3 and $5 \mathrm{~m}$. Figure 4 shows examples of the $2 \mathrm{D}$ map when the accumulated times are $0.1,0.3$ and 0.5 s. In Figs. 3 and 4, the center point on each map corresponds to the TER position, and the other points represent the positions of obstacles.

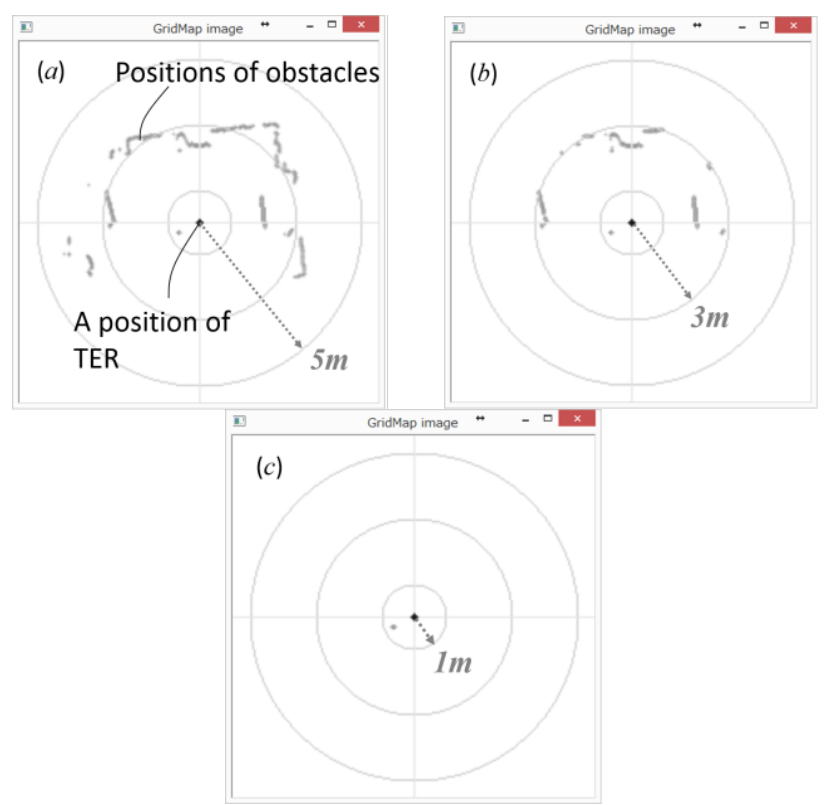

Figure 3: Real-time blurred 2D maps when the scan range is: (a) $5 \mathrm{~m}$, (b) $3 \mathrm{~m}$, (c) $1 \mathrm{~m}$ 


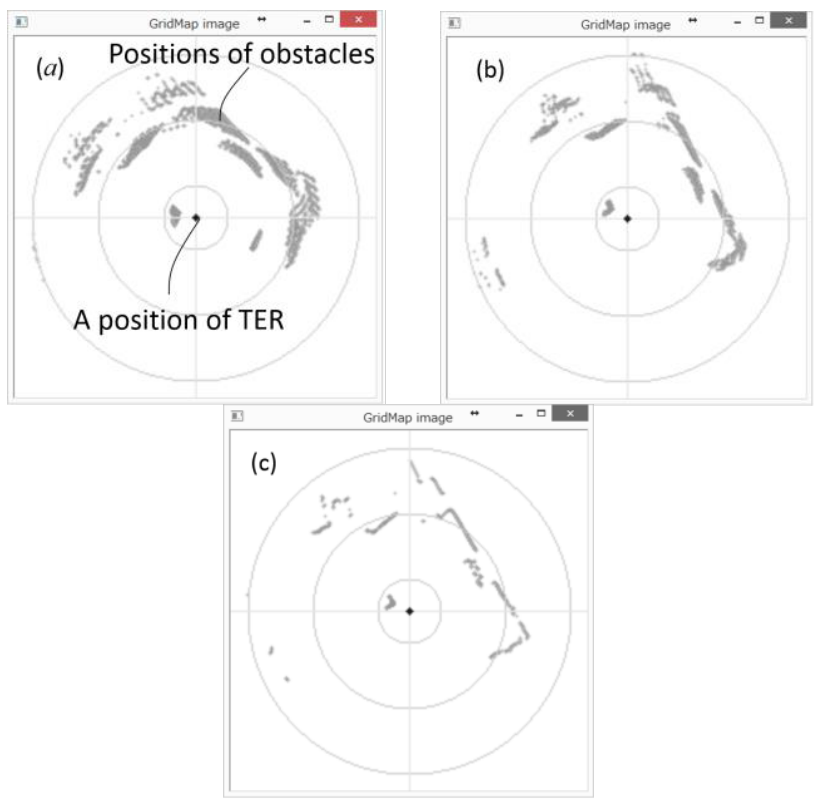

Figure 4: Real-time blurred 2D maps for accumulated times: (a) $0.5 \mathrm{~s}$, (b) $0.3 \mathrm{~s}$, (c) $0.1 \mathrm{~s}$

In the case of a lasers scan range, more plots are displayed, as shown in Fig.3. The longer the accumulated time, the stronger the motion blur displayed as shown in Fig.4.

\section{Tele-operation Experiment to Investigate Spatial Perception}

\subsection{Experimental conditions}

The content and procedure of the experiment were approved by the Tokyo Denki University human bioethics review committee, and the experiment was only conducted after explaining it to the participant and receiving participant approval. A male aged 24 years participated in the experiment.

Human spatial perception was investigated by an experiment using the tele-operation system, as shown in Fig.2. In the experiment, the participant manipulated the TER around the obstacle course shown in Figure 5. A scan range 1,2 or $3 \mathrm{~m}$ and an accumulated time of $0.1,0.3$ or $0.5 \mathrm{~s}$ were used; and the tasks was repeated until all conditions (nine cases) has been employed.

In each task, we measured distances between the TER and the obstacles by the LRF, and human spatial perception was investigated by analyzing the minimum distance measured by the LRF. First, factor 1 was examined by performing a Kruskal-Wallis test with a Wilcoxon signed-rank test against the average minimum distance for each task with the same scan range. Second, factor 2 was examined by statistical analysis against the average minimum distance for each task with the same accumulated time.

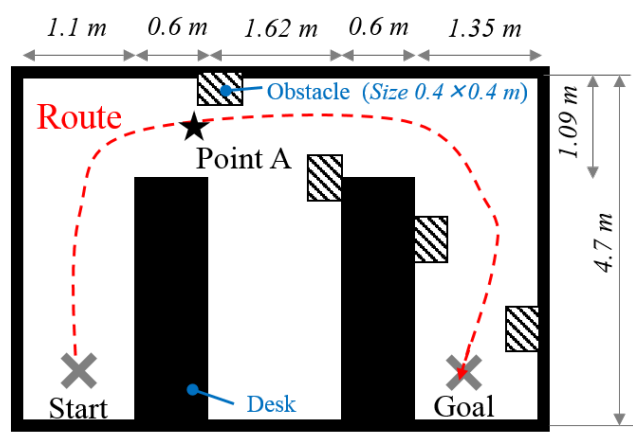

Figure 5: Overview of experimental course

\subsection{Experimental results}

It is difficult to recognize the position of obstacles after curve, like the obstacle in the vicinity of point A. Also, the user will be easily influenced by changes in the scan range. Thus, factor 1 was investigated using the distance data obtained during system manipulation from the start point to point A. The number of sampled LRF distances was 200. On the other hand, the motion blur is expected to influence the performance of the subject in the experiment; hence, factor 2 was investigated using all the distance data from the start point to the goal. The number of sampled LRF distances was 600.

First, the minimum distances the same scan range were compared using the Kruskal-Wallis test, and significant differences in the minimum distance were confirmed when the scan range in the radar map was changed among 1,3 and $5 \mathrm{~m}(H(599)=41.75, \quad p<.001,95 \%)$. Next, another statistical analysis was conducted using Wilcoxon signed-rank test for the three cases with scan range. Figure 6(a) shows the average minimum distance for scan ranges the 1, 3 and $5 \mathrm{~m}$. The error bar represents standard error. From Fig.6(a), the minimum distance increased as the scan range increased during system manipulation. It was inferred that the operator could better understand the positional relation of obstacles around the robot by increasing the scan range; because the map helped the operator to recognize positional relations [9].

Second, the minimum distance was compared among the cases with the same accumulated times using the Kruskal- Wallis test, and significant differences in the minimum distance were confirmed when the accumulated time was changed among $0.1,0.3$ and $0.5 \mathrm{~s}$ $(H(1799)=39.21, p<.001,95 \%)$. Further analysis was conducted using the Wilcoxon signed-rank test for the three cases with each accumulated time. Fig.6(b) shows 

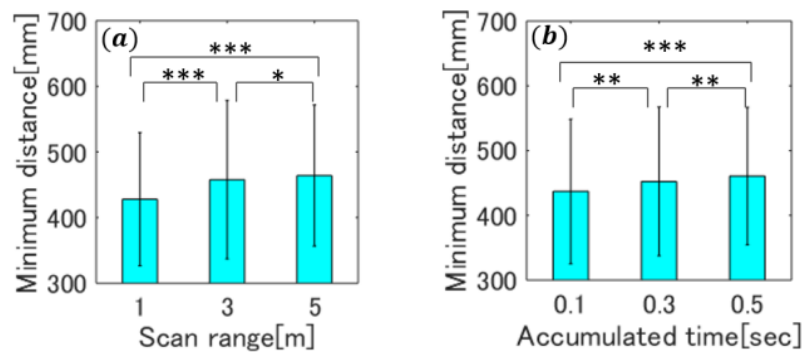

Figure 6: Averages of minimum distance $(*: p<.05$,

${ }^{* *}: p<.01,{ }^{* * *}: p<.001$; Wilcoxon signed-rank test):

(a) Scan range, (b) Accumulated time

the average minimum distance for accumulated times of $0.1,0.3$ and $0.5 \mathrm{~s}$. From Fig.6(b), the minimum distance increased as the accumulated time increased. It was inferred that the operator could understand the motion of the robot from the motion blur, since the motion blur improved the perception of motion.

From Figs.5 and 6, it is summarized that the expansion of the scan range helped the operator to recognize a location during manipulation and that increasing the accumulated time for the blur on the radar map helped the operator to recognize the movement of the robot. Therefore, it is concluded that the operator's spatial perception at a remote locations was improved by be increasing of the scan range and the motion blur on the map. In short, the real-time blurred 2D map with $5 \mathrm{~m}$ and $0.5 \mathrm{~s}$ as the presentation specifications was best among the nine conditions.

\section{Conclusion}

In this paper, a new remote manipulation system providing a real-time blurred 2D map was prototyped, and the most suitable conditions for the blurred 2D map were investigated by a tele-operation experiment. The operational performance was investigated by analyzing distances measured by the LRF during TER manipulation.

Significant differences in the minimum distance were confirmed when the scan range in the radar map was changed among 1,3 and $5 \mathrm{~m}(H(599)=41.75, \quad p<.001$, $95 \%$ ). Also, significant differences in the minimum distance were confirmed when the accumulated time was changed among $0.1,0.3$ and $0.5 \mathrm{~s}(H(1799)=39.21, p<.001$, $95 \%)$. Therefore, the operator's spatial perception at a remote locations was improved by increasing the scan range and the motion blur on the map. In the future, we would like to investigate the operator's spatial perception in the case of a longer range of the map because we are not find a suboptimal solution of the conditions of the map for the operator's spatial perception. Also, in order to confirm individual differences for the results of this experiment, we would like to conduct this experiment with multiple participants.

\section{Acknowledgments}

This research was partly supported by a JSPS Grant-inAid for Scientific Research (C) (Grant Number 15K06153), Japan. The authors wish to thank JSPS for their generous financial assistance and many participants who cooperated in our experiments.

\section{References}

[1] K. Yanasawa, Y. Onoe, N. Yokoya and H. Takemura: Telepresence by real-time view-dependent image generation from omnidirectional images, ICICE Transactions on Fundamentals of Electronics, Vol .J81D-1, No. 5 , pp. 880-887, 1998.

[2] M. Imai, et al.: Avatar robot system using omnidirection trolley, Conference on Cloud Network Robot Service, Vol. 112, No. 436, pp. 27-30, 2013.

[3] S. Kratz, J. Vaughan and D. Kimber: Evaluating stereoscopic video with head tracking for immersive teleoperation of mobile telepresence robots, ACM/IEEE International Conference on Human-Robot Interaction (HRI), pp. 43-44, 2015.

[4] D. Letourneau et al.: Exploratory design and evaluation of a homecare teleassistive mobile robotic system, Mechatronics, Vol. 20, No. 7, pp. 751-766, 2010.

[5] H. Head and G. Holmes: Sensory disturbance from cerebral lesions, Brain, Vol. 34, pp. 102-254, 1911.

[6] T. Pfeiffer et al.: Stereo vision and acuity tests within a virtual reality Set-Up, 10. Workshop der GIFachgruppe VR/AR, pp. 185-188, 2013.

[7] W. Yoshiki: The role of maps in the cognition of geographic space, Cognitive Studies: Bulletin of the Japanese Cognitive Science Society, Vol. 15, No. 1, pp. 38-50, 2008.

[8] K. Imap, M. Mukunoki and K. Ikeda: Improvement of motion perception with blur in scroll screen, Pattern Recognition and Media Understanding (PRMU), Vol. 97, No. 386, pp. 155-160, 1997.

[9] K. Fujii, S. Azuma and K. Arai: The influence of route guide on navigation process, ICICE Transactions on Fundamentals of Electronics, Vol. 87-A, No. 1, pp. 40-49, 2004. 